\title{
Bederite, a new pegmatite phosphate mineral from Nevados de Palermo, Argentina: Description and crystal structure
}

\author{
Miguel A. Galliski,* Mark A. Cooper, Frank C. Hawthorne, $\dagger$ ANd Petr Černý
}

Department of Geological Sciences, University of Manitoba, Winnipeg, Manitoba, Canada R3T 2N2

\begin{abstract}
Bederite, ideally $\square \mathrm{Ca}_{2} \mathrm{Mn}_{2}^{2+} \mathrm{Fe}_{2}^{3+} \mathrm{Mn}_{2}^{2+}\left(\mathrm{PO}_{4}\right)_{6}\left(\mathrm{H}_{2} \mathrm{O}\right)_{2}$, orthorhombic, $a=12.559(2), b=12.834(1), c$ $=11.714(2) \AA, V=1887.8(4) \AA^{3}, Z=4$, space group $P c a b$, is a new mineral from the El Peñón pegmatite, Nevados de Palermo, Salta Province, República Argentina. The mineral occurs as rare ellipsoidal nodules ( $\sim 5 \mathrm{~cm}$ in diameter) enclosed in potassium feldspar or quartz at the core-margin zone of a beryl-type rare-element pegmatite. Associated minerals are quartz, potassium feldspar, muscovite, beryl, columbite, possibly heterosite, and powdery coatings of $\mathrm{Mn}$ - and Fe-oxides; in the dumps of the pegmatite, there are numerous other phosphates including altered triphylite-lithiophyllite, arrojadite, eosphorite, laueite, brazilianite, and fairfieldite. Bederite is very dark brown to black with a dark olive-green streak and a vitreous luster. It is brittle with an irregular fracture and a good cleavage parallel to $\{100\}$, Mohs hardness is 5, and the observed and calculated densities are 3.48(1) and $3.50 \mathrm{~g} / \mathrm{cm}^{3}$, respectively. In transmitted plane-polarized light, bederite is pleochroic $\mathrm{X}=\mathrm{Y}=$ olive green, $\mathrm{Z}=$ brown with $\mathrm{X}=\mathrm{Y}>\mathrm{Z}$ and $\mathrm{X}=a, \mathrm{Y}=c, \mathrm{Z}=b$. In cross-polarized light, it is biaxial negative with strong dispersion, $v>r, 2 \mathrm{~V}(\mathrm{obs})=54^{\circ}$ and $2 \mathrm{~V}$ (calc) $=60^{\circ}$. Refractive indices are as follows: $\alpha=1.729(3), \beta=1.738(3), \gamma=1.741(3)$. Chemical analysis by electron microprobe plus the Penfield method and thermogravimetry gave $\mathrm{P}_{2} \mathrm{O}_{5} 41.76, \mathrm{Al}_{2} \mathrm{O}_{3} 0.82, \mathrm{Fe}_{2} \mathrm{O}_{3} 12.00, \mathrm{FeO} 2.25, \mathrm{MnO}$ 20.59, $\mathrm{MgO} 3.45, \mathrm{ZnO} 0.40, \mathrm{CaO} 10.91, \mathrm{SrO} 0.43, \mathrm{Na}_{2} \mathrm{O} 0.63, \mathrm{H}_{2} \mathrm{O} 3.52$, sum 96.76 wt $\%$ where the $\mathrm{Fe}_{2} \mathrm{O}_{3}$ and $\mathrm{FeO}$ contents were derived from the refined crystal structure. The five strongest lines in the $\mathrm{X}$-ray powder diffraction pattern are as follows: $d(\AA), I,(h k l): 2.768,100,(402) ; 2.927,78,(004)$; 3.006, 67, (1 4 1); 2.814, 35, (0 4 2); 2.110, 33, (1 60 ). The crystal structure of bederite was refined to an $R$ index of $2.8 \%$ based on 2530 observed $(>5 \sigma \mathrm{F})$ reflections measured with MoK $\alpha \mathrm{X}$-radiation. Bederite is isostructral with wicksite, grischunite, and an unnamed wicksite-like phase; it is related to wicksite by the substitutions ${ }^{\mathrm{Na}} \square+{ }^{\mathrm{M} 2} \mathrm{Fe}^{3+} \rightarrow{ }^{\mathrm{Na}} \mathrm{Na}+{ }^{\mathrm{M} 2} \mathrm{Mg},{ }^{\mathrm{M} 1} \mathrm{Mn}^{2+} \rightarrow{ }^{\mathrm{M} 1} \mathrm{Fe}^{2+}$ and ${ }^{\mathrm{M} 3} \mathrm{Mn}^{2+} \rightarrow{ }^{\mathrm{M} 3} \mathrm{Fe}^{2+}$.
\end{abstract}

\section{INTRODUCTION}

Galliski and Sureda (1982) reported nodules of a phosphate mineral that they classified generically as a member of the alluaudite group. The X-ray diffraction pattern was similar to that of wicksite (Sturman et al. 1981), but wet-chemical analysis (Galliski and Sureda 1982) gave a significantly different chemical composition, with $\mathrm{MnO}$ dominant over $\mathrm{FeO}$, and suggested strong similarities with the unnamed wicksite-like phase of Peacor et al. (1985). Solution of the crystal structure of the phase showed that it is isostructural but chemically distinct from wicksite. The Commission of New Minerals and Mineral Names of the International Mineralogical Association has approved the mineral and the name: bederite. The name honors Roberto Beder (born in Basel, Switzerland and died in Córdoba, Argentina: 1888-1930) for his major contribution to the development of Mineralogy in Argentina, particularly his work in morphological crystallography. Holotype and cotype samples

*Current address: IANIGLA-CONICET, Centro Regional de Investigaciones Cientificas y Tecnicas, Avda. Adrian Ruiz Leal, Parque General San Martin, (5500) Mendoza, Argentina †E-mail: Frank_hawthorne@umanitoba.ca of bederite are deposited at the Canadian Museum of Nature, Ottawa (CMNMC 81556) and in the Museo Prof. M. Tellechea, CRICYT, Mendoza (no. 8553).

\section{OCCURRENCE}

Bederite occurs in the El Peñón pegmatite, located approximately at $24^{\circ} 50, \mathrm{~S}, 66^{\circ} 20$, W, $3920 \mathrm{~m}$ above sea level in the El Quemado pegmatite field, Nevados de Palermo, Salta Province, República Argentina. El Quemado is the northernmost pegmatite field of the Pampean Pegmatite Province. Galliski (1983a, 1983b) described the geology of the area and its pegmatites. El Peñón is a rare-element pegmatite of the beryl type, beryl-columbite-phosphate subtype, which was discordantly emplaced in mottled cordierite-bearing micaschists. It is $60 \mathrm{~m}$ long and from 4 to $10 \mathrm{~m}$ wide, is approximately tabular, and strikes $\mathrm{N} 40-45^{\circ} \mathrm{W}$ with a dip of $65^{\circ}$ to the southwest. The pegmatite consists of border, wall and intermediate zones, a core zone, and replacement units. The border zone is $5 \mathrm{~cm}$ wide, with fine-grained quartz, plagioclase $\left(\mathrm{An}_{14-18}\right)$, and muscovite as the principal minerals, and apatite and schorl as accessory phases. The wall zone is medium-grained, with quartz, plagioclase $\left(\mathrm{An}_{8-10}\right)$, potassium feldspar, and muscovite as the principal minerals. The wall zone grades into the intermediate 
zone, which has the same mineralogy but is much coarser grained. The core is composed of massive milky quartz. The main accessory minerals are concentrated in the core-margin zone and in the replacement units, and do not represent more than $1-2 \%$ of the pegmatite; they include white-yellowish beryl, tourmaline, fine-grained muscovite, bismuthinite, columbite, tantalite, uraninite, Mn-oxides, microlite, tapiolite, triphylitelithiophylite, eosphorite, brazilianite, arrojadeite, bederite, fairfieldite, phosphosiderite, and laueite. The pegmatite was exploited for tantalite in 1943-1945.

\section{PhySICAL PROPERTIES}

Bederite occurs as rare, massive ellipsoidal nodules up to $\sim 5 \mathrm{~cm}$ in diameter and encased in potassium feldspar or quartz in the wall of the main quarry. In situ, the nodules are dark and resemble minerals of the columbite group, from which they are easily distinguished by their low specific gravity. The surfaces of the nodules are partly covered by flakes of muscovite, and the nodules are crosscut by veinlets of microcrystalline quartz. On broken surfaces of the nodules, cleavage planes are prominent and exhibit a marked radial arrangement. Bederite is very dark brown to black with a dark olive-green streak; thin fragments show a range of colors from brown to olive green. It has a vitreous luster and does not fluoresce under ultraviolet light. It is brittle with an irregular fracture and a good cleavage parallel to $\{100\}$. Mohs hardness is 5 , and the measured and calculated densities are $3.48(1)$ and $3.50 \mathrm{~g} / \mathrm{cm}^{3}$, respectively (measured with a Berman torsion balance).

\section{OPTICAL PROPERTIES}

In transmitted light, bederite is pleochroic $\mathrm{X}=\mathrm{Y}=$ olive green, $\mathrm{Z}=$ brown with $\mathrm{X}=\mathrm{Y}>\mathrm{Z}$ and optical orientation $\mathrm{X}=a$, $\mathrm{Y}=c, \mathrm{Z}=b$. In cross-polarized light, bederite is biaxial negative with strong dispersion, $v>r, 2 \mathrm{~V}$ (obs.) $=54^{\circ}$ and $2 \mathrm{~V}$ (calc.) $=60^{\circ}$. The mineral is normally optically homogeneous, except on $\{100\}$ where small optical domains with imperfect and variable extinction (resembling tartan twinning in microcline) are observed locally. Refractive indices were measured in monochromatic light $(\lambda=589 \mathrm{~nm})$, giving the following values: $\alpha=$ 1.729(3), $\beta=1.738(3), \gamma=1.741(3)$.

\section{CHEMICAL COMPOSITION}

Crystals were analyzed with a Cameca SX-50 electron-microprobe operating in wavelength-dispersion mode with an accelerating voltage of $15 \mathrm{kV}$, a specimen current of $20 \mathrm{nA}$, a beam size of $5 \mu \mathrm{m}$, and counting times on peak and background of 20 and 10 s, respectively. The following standards were used: albite $(\mathrm{Na})$, fayalite $(\mathrm{Fe})$, apatite $(\mathrm{Ca}, \mathrm{P})$, pyrope $(\mathrm{Mg}, \mathrm{Al})$, spessartine $(\mathrm{Mn}), \mathrm{SrTiO}_{3}(\mathrm{Sr})$, and $\mathrm{ZnS}(\mathrm{Zn})$. Data were reduced using the $\phi(\rho Z)$ method of Pouchou and Pichoir (1985). Table 1 gives the mean of 9 analyses from the crystal used for structure determination, together with the range of composition for 39 points on 5 different fragments of the same nodule. The $\mathrm{Fe}^{3+} / \mathrm{Fe}^{2+}$ ratio was derived from the crystal-structure analysis; $\mathrm{H}_{2} \mathrm{O}$ was measured by the Penfield method and confirmed by thermogravimetry and by crystal-structure analysis. The sum of the oxides is significantly less than $100 \%$. This feature is probably due to slight charging of the analyzed crystal; in our experience, such low totals are fairly typical of analyzing small crystals embedded in epoxy; simultaneous analysis of large crystals gives sums $\sim 100 \%$. The chemical formula was calculated on the basis of $26 \mathrm{O}$ atoms, (Table 1) and assigned to different groups so as to be conformable with the refined sitescattering values.

\section{X-RAY DIFFRACTION}

\section{Powder diffraction}

The X-ray powder-diffraction pattern was recorded on a Siemens D5000 diffractometer using monochromatic $\mathrm{CuK \alpha} \alpha_{1}$ radiation and calibrated against NBS Si-640b.

\section{Single-crystal diffraction}

Careful optical examination in non-polarized, polarized, and cross-polarized light identified a single crystal of good quality, and this was then ground to an ellipsoid of diameter $\sim 0.24 \mathrm{~mm}$ using a Bond sphere-grinder. The crystal was mounted on a Siemens $P 4$ automated four-circle diffractometer and aligned on 41 relatively intense reflections from 35 to $60^{\circ} 2 \theta$. The orientation matrix and cell dimensions were determined from the setting angles by least-squares refinement; the cell dimensions are given in Table 3. A total of 7363 reflections was collected over the index ranges $\overline{2} \leq h \leq 17, \overline{2} \leq k \leq 18, \overline{2} \leq l \leq 16$, with scan speeds between 3.0 and $30.0^{\circ} 2 \theta / \mathrm{min}$ and over the range $4 \leq 2 \theta \leq 60^{\circ}$. Subsequent to the $\theta-2 \theta$ data-collection, psi-scan data were collected for 17 reflections at $4^{\circ}$ intervals of psi. The psi-scan data were corrected for absorption, modeling the crystal as an ellipsoid and reducing $R$ (azimuthal) from 3.9 to $1.2 \%$. The $\theta-2 \theta$ intensity data were corrected for absorption, Lorentz, TABLE 1. Chemical composition* (wt\%) and unit formula (apfu) of

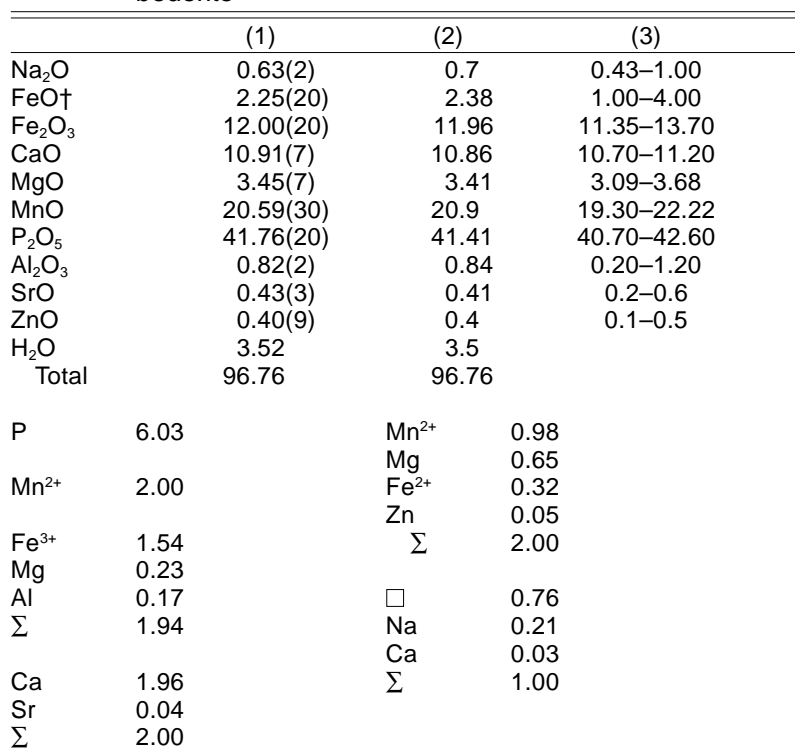

Notes: (1) Crystal used for structure study, mean of nine determinations and normalized to the same total as column $1 ;(2)$ values calculated from the refined structure; (3) range of 39 determinations on 5 different crystal fragments from the same nodule.

${ }^{*} \mathrm{~K}, \mathrm{Cu}, \mathrm{F}, \mathrm{Ti}, \mathrm{Si}, \mathrm{As}, \mathrm{V}, \mathrm{Cr}, \mathrm{S}$, and $\mathrm{Pb}$ below detection.

$\dagger \mathrm{Fe}^{2+}$ and $\mathrm{Fe}^{3+}$ contents determined by SREF. 
TABLE 2. X-ray powder-diffraction data for bederite

\begin{tabular}{|c|c|c|c|c|}
\hline$l_{\text {obs }}$ & $d_{o b s}$ & $d_{\text {calc }}$ & $I_{\text {calc }}$ & $h k l$ \\
\hline 4 & 7.116 & 7.126 & 3 & 111 \\
\hline 31 & 6.419 & 6.417 & 14 & $020^{*}$ \\
\hline 23 & 6.284 & 6.283 & 19 & 200 * \\
\hline 30 & 5.853 & 5.856 & 18 & 002 * \\
\hline 2 & 5.337 & 5.328 & 2 & 012 \\
\hline 2 & 5.138 & 5.136 & 1 & 121 \\
\hline 2 & 4.904 & 4.905 & 1 & 112 \\
\hline 24 & 4.491 & 4.489 & 17 & $220^{*}$ \\
\hline 13 & 4.326 & 4.326 & 10 & $022^{*}$ \\
\hline 5 & 4.192 & 4.192 & 4 & $221^{*}$ \\
\hline 6 & 4.062 & 4.064 & 5 & $212^{*}$ \\
\hline 14 & 3.829 & 3.828 & 12 & $131^{*}$ \\
\hline 10 & 3.564 & 3.563 & 9 & $222^{*}$ \\
\hline 23 & 3.507 & 3.508 & 19 & $320^{*}$ \\
\hline 5 & 3.336 & 3.331 & 3 & 132 \\
\hline 7 & 3.031 & 3.034 & 4 & 401 \\
\hline 67 & 3.006 & 3.005 & 59 & $141^{*}$ \\
\hline 9 & 2.9509 & 2.9529 & 5 & 411 \\
\hline \multirow[t]{2}{*}{78} & 2.9274 & 2.9281 & 85 & $004^{*}$ \\
\hline & & 2.8576 & 16 & $240^{*}$ \\
\hline \multirow[t]{2}{*}{31} & 2.8559 & & & \\
\hline & & 2.8547 & 16 & $014^{*}$ \\
\hline \multirow[t]{2}{*}{35} & 2.8142 & 2.8139 & 31 & $042^{*}$ \\
\hline & & 2.7838 & 5 & 114 \\
\hline \multirow[t]{2}{*}{100} & 2.7682 & & & \\
\hline & & 2.7683 & 100 & $402^{*}$ \\
\hline 29 & 2.7434 & 2.7431 & 25 & $421^{*}$ \\
\hline \multirow[t]{2}{*}{15} & 2.7058 & 2.7061 & 14 & $412^{*}$ \\
\hline & & 2.6639 & 2 & 024 \\
\hline \multirow[t]{2}{*}{4} & 2.6572 & & & \\
\hline & & 2.6540 & 3 & 204 \\
\hline 16 & 2.5683 & 2.5681 & 16 & $242^{*}$ \\
\hline 6 & 2.5420 & 2.5419 & 4 & $422^{*}$ \\
\hline 21 & 2.4891 & 2.4890 & 19 & $341^{*}$ \\
\hline 13 & 2.4587 & 2.4589 & 10 & $151^{*}$ \\
\hline 14 & 2.4329 & 2.4320 & 13 & $143^{*}$ \\
\hline 4 & 2.4134 & 2.4134 & 3 & $511^{*}$ \\
\hline 8 & 2.3405 & 2.3401 & 6 & $520^{*}$ \\
\hline 6 & 2.3248 & 2.3242 & 5 & $432^{*}$ \\
\hline 7 & 2.2655 & 2.2666 & 5 & 115 \\
\hline 2 & 2.2446 & 2.2447 & 2 & 440 \\
\hline 2 & 2.2014 & 2.2019 & 1 & 252 \\
\hline 4 & 2.1625 & 2.1635 & 3 & 215 \\
\hline \multirow[t]{2}{*}{3} & 2.1518 & 2.1514 & 3 & 351 \\
\hline & & 2.1143 & 5 & 153 \\
\hline \multirow[t]{2}{*}{33} & 2.1100 & & & \\
\hline & & 2.1088 & 23 & 160 \\
\hline 13 & 2.0942 & 2.0943 & 7 & $600^{*}$ \\
\hline 3 & 2.0621 & 2.0616 & 3 & 601 \\
\hline 10 & 2.0447 & 2.0451 & 10 & $244^{*}$ \\
\hline 2 & 2.0188 & 2.0190 & 2 & 315 \\
\hline 3 & 1.9841 & 1.9840 & 3 & 162 \\
\hline \multirow[t]{2}{*}{7} & 1.9725 & 1.9720 & 7 & $602^{*}$ \\
\hline & & 1.9521 & 3 & 006 \\
\hline \multirow[t]{3}{*}{6} & 1.9502 & & & \\
\hline & & 1.9509 & 2 & 541 \\
\hline & & 1.9091 & 7 & 353 \\
\hline \multirow[t]{2}{*}{10} & 1.9079 & & & \\
\hline & & 1.9075 & 4 & 116 \\
\hline 2 & 1.8847 & 1.8850 & 2 & 622 \\
\hline 2 & 1.8712 & 1.8709 & 3 & 145 \\
\hline 4 & 1.8565 & 1.8572 & 3 & 631 \\
\hline & & 1.8473 & 6 & 126 \\
\hline 10 & 1.8454 & & & \\
\hline & & 1.8448 & 5 & 216 \\
\hline 4 & 1.8277 & 1.8281 & 5 & $524^{*}$ \\
\hline 3 & 1.8019 & 1.8023 & 3 & 425 \\
\hline 4 & 1.7927 & 1.7929 & 2 & $171^{*}$ \\
\hline 9 & 1.7815 & 1.7815 & 9 & $444^{*}$ \\
\hline 10 & 1.7538 & 1.7538 & 10 & $640^{*}$ \\
\hline & & 1.7288 & 4 & 720 \\
\hline
\end{tabular}

TABLE 2.-Continued

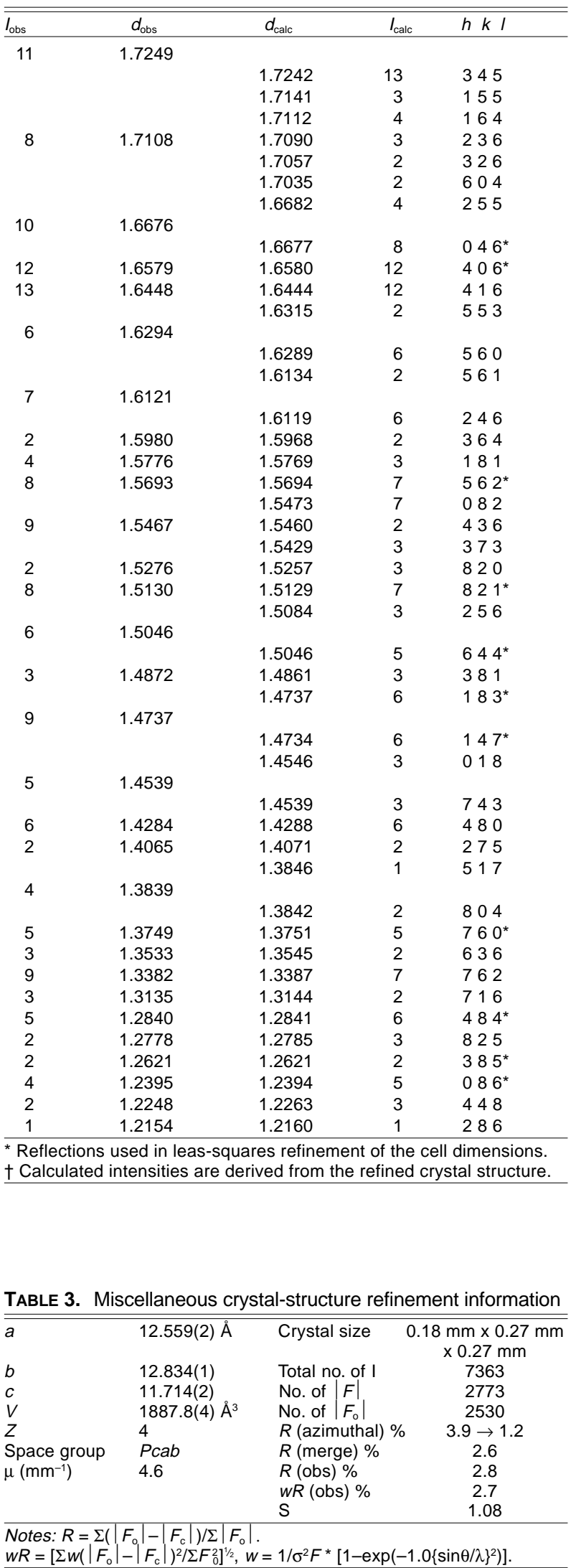


TABLE 4. Atom positions and displacement parameters $\left(\times 10^{4}\right)$ for bederite

\begin{tabular}{|c|c|c|c|c|c|c|c|c|c|c|}
\hline & $x$ & $y$ & $z$ & $U_{11}$ & $U_{22}$ & $U_{33}$ & $U_{23}$ & $U_{13}$ & $U_{12}$ & $U_{\text {eq }}$ \\
\hline $\mathrm{P} 1$ & $0.38715(4)$ & $0.29998(4)$ & $0.23044(4)$ & $93(2)$ & $71(2)$ & $61(2)$ & $-8(2)$ & $-4(2)$ & $2(2)$ & $75(1)$ \\
\hline P2 & $0.09661(4)$ & $0.44479(4)$ & $0.25952(4)$ & 104(2) & $72(2)$ & $73(2)$ & $4(2)$ & $-11(2)$ & $-2(2)$ & $83(1)$ \\
\hline P3 & $0.26392(4)$ & $0.12462(4)$ & $0.47847(4)$ & 137(2) & $70(2)$ & $69(2)$ & $0(2)$ & $-1(2)$ & $-5(2)$ & $92(1)$ \\
\hline M1 & $0.14793(3)$ & $0.20846(3)$ & $0.23362(3)$ & 119(2) & $87(2)$ & $128(2)$ & $20(1)$ & $13(1)$ & $9(1)$ & 111(1) \\
\hline M2 & $0.33024(3)$ & $0.04152(3)$ & $0.22862(2)$ & $93(2)$ & $68(2)$ & $62(2)$ & $-4(1)$ & $0(1)$ & $-5(1)$ & $75(1)$ \\
\hline M3' & $0.0098(6)$ & $0.2840(10)$ & $0.4646(1)$ & & & & & & & $89(5)$ \\
\hline M3" & $-0.0036(4)$ & $0.3062(7)$ & $0.4655(1)$ & & & & & & & $110(7)$ \\
\hline M3"' & $0.0247(6)$ & $0.2644(7)$ & $0.4611(2)$ & & & & & & & $99(7)$ \\
\hline $\mathrm{Na}$ & $0.0090(9)$ & $-0.0085(9)$ & $-0.0162(7)$ & & & & & & & 261(30) \\
\hline $\mathrm{Ca}$ & $0.26053(3)$ & $0.37624(3)$ & $0.49935(3)$ & $139(2)$ & $98(2)$ & $88(1)$ & $13(1)$ & 1(1) & $-15(1)$ & $108(1)$ \\
\hline 01 & $0.4925(1)$ & $0.2729(1)$ & $0.2894(1)$ & $119(6)$ & $157(7)$ & $117(5)$ & $-4(5)$ & $-26(5)$ & $25(6)$ & 131(4) \\
\hline $\mathrm{O} 2$ & $0.4053(1)$ & $0.3370(1)$ & $0.1078(1)$ & $177(7)$ & $137(7)$ & $75(5)$ & 11(5) & $13(5)$ & $14(6)$ & $130(4)$ \\
\hline O3 & $0.3171(1)$ & $0.2010(1)$ & $0.2272(1)$ & $137(6)$ & $85(6)$ & $165(6)$ & $-16(5)$ & $-8(5)$ & $-22(5)$ & $129(4)$ \\
\hline O4 & $0.3301(1)$ & $0.3849(1)$ & $0.3012(1)$ & $120(6)$ & $114(6)$ & $108(5)$ & $-30(5)$ & $10(5)$ & $3(5)$ & 114(3) \\
\hline O5 & $0.1637(1)$ & $0.5455(1)$ & $0.2561(1)$ & $156(6)$ & $75(6)$ & $127(5)$ & $3(5)$ & $-2(5)$ & $-17(5)$ & $119(3)$ \\
\hline O6 & $0.1489(1)$ & $0.3637(1)$ & $0.1815(1)$ & $142(7)$ & $95(6)$ & $132(6)$ & $-31(5)$ & $2(5)$ & $14(5)$ & $123(4)$ \\
\hline O7 & $0.0918(1)$ & $0.4077(1)$ & $0.3839(1)$ & $211(7)$ & $149(7)$ & $95(5)$ & $37(5)$ & $-29(5)$ & $-51(6)$ & $152(4)$ \\
\hline O8 & $-0.0148(1)$ & $0.4678(1)$ & $0.2101(1)$ & $117(6)$ & $179(7)$ & $163(6)$ & $-4(6)$ & $-29(5)$ & $29(6)$ & $153(4)$ \\
\hline 09 & $0.1891(1)$ & $0.2041(1)$ & $0.4213(1)$ & $205(7)$ & $97(6)$ & $110(5)$ & $10(5)$ & $2(5)$ & $32(6)$ & $137(4)$ \\
\hline 010 & $0.3503(1)$ & $0.1893(1)$ & $0.5413(1)$ & 194(7) & $150(7)$ & $122(5)$ & $-21(5)$ & $-4(6)$ & $-70(6)$ & $155(4)$ \\
\hline 011 & $0.1997(1)$ & $0.0522(1)$ & $0.5579(1)$ & $179(7)$ & $109(6)$ & $106(5)$ & $16(5)$ & $-6(5)$ & $-32(6)$ & 131(4) \\
\hline 012 & $0.3227(1)$ & $0.0493(1)$ & $0.3971(1)$ & $235(8)$ & $156(7)$ & $96(5)$ & $-16(5)$ & $-19(6)$ & $65(6)$ & $162(4)$ \\
\hline 013 & $-0.0279(2)$ & $0.1184(2)$ & $0.4910(1)$ & $213(9)$ & 283(9) & $148(6)$ & $62(6)$ & $10(6)$ & $46(7)$ & $215(5)$ \\
\hline $\mathrm{H} 1$ & $0.003(4)$ & $0.082(4)$ & $0.557(3)$ & & & & & & & $539(97)$ \\
\hline $\mathrm{H} 2$ & $0.002(4)$ & $0.081(4)$ & $0.425(3)$ & & & & & & & $539(97)$ \\
\hline
\end{tabular}

TABLE 5. Selected interatomic distances $(\AA ̊)$ in bederite

\begin{tabular}{|c|c|c|c|}
\hline $\mathrm{P} 1-\mathrm{O} 1$ & $1.533(2)$ & M1-O1d & $2.072(2)$ \\
\hline $\mathrm{P} 1-\mathrm{O} 2$ & $1.530(1)$ & M1-O3 & $2.128(2)$ \\
\hline $\mathrm{P} 1-\mathrm{O} 3$ & $1.546(2)$ & M1-05a & $2.104(2)$ \\
\hline P1-O4 & $1.545(2)$ & M1-O6 & $2.084(2)$ \\
\hline$<\mathrm{P} 1-\mathrm{O}\rangle$ & 1.539 & $\begin{array}{l}\text { M1-O9 } \\
M 1-010 c\end{array}$ & $\begin{array}{l}2.259(1) \\
2.267(2)\end{array}$ \\
\hline P2-O5 & $1.543(2)$ & $<\mathrm{M} 1-\mathrm{O}>$ & 2.152 \\
\hline P2-O6 & $1.533(2)$ & & \\
\hline P2-O7 & $1.534(1)$ & M2-O3 & $2.053(2)$ \\
\hline P2-O8 & $1.542(2)$ & M2-O4a & $2.040(2)$ \\
\hline \multirow[t]{2}{*}{$<\mathrm{P} 2-\mathrm{O}>$} & 1.538 & M2-O5a & $2.100(2)$ \\
\hline & & $\mathrm{M} 2-\mathrm{O} 8 \mathrm{~b}$ & $1.962(2)$ \\
\hline P3-O9 & $1.540(2)$ & M2-O11c & $2.039(1)$ \\
\hline P3-O10 & $1.551(2)$ & M2-O12 & $1.979(1)$ \\
\hline P3-011 & $1.543(2)$ & $<\mathrm{M} 2-\mathrm{O}>$ & 2.029 \\
\hline P3-012 & $1.545(2)$ & & \\
\hline \multirow[t]{2}{*}{$<\mathrm{P} 3-\mathrm{O}>$} & 1.545 & M3'-O1d & $2.190(5)$ \\
\hline & & M3'-O2e & $2.101(6)$ \\
\hline $\mathrm{Na}-\mathrm{O} 2 \mathrm{f}$ & $2.498(11)$ & M3'-O7 & $2.116(10)$ \\
\hline $\mathrm{Na}-\mathrm{O} 2 \mathrm{~g}$ & $2.941(11)$ & M3'-O9 & $2.526(9)$ \\
\hline $\mathrm{Na}-\mathrm{O} 7 \mathrm{a}$ & $2.155(10)$ & M3'-O10d & $2.222(8)$ \\
\hline $\mathrm{Na}-\mathrm{O} 7 \mathrm{~h}$ & $2.155(10)$ & M3'-O13 & $2.199(12)$ \\
\hline $\mathrm{Na}-\mathrm{O} 12 \mathrm{i}$ & $2.774(10)$ & $<\mathrm{M}^{\prime}-\mathrm{O}>$ & 2.226 \\
\hline $\mathrm{Na}-\mathrm{O} 12 \mathrm{c}$ & $2.459(11)$ & & \\
\hline \multirow[t]{2}{*}{$<\mathrm{Na}-\mathrm{O}>$} & 2.497 & M3"-O1d & $2.300(5)$ \\
\hline & & M3"-O2e & $2.111(4)$ \\
\hline $\mathrm{Ca}-\mathrm{O} 2 \mathrm{e}$ & $2.490(2)$ & M3"-O7 & $2.011(7)$ \\
\hline $\mathrm{Ca}-\mathrm{O} 4$ & $2.483(2)$ & M3"-O9 & $2.800(6)$ \\
\hline $\mathrm{Ca}-\mathrm{O} 6 \mathrm{e}$ & $2.423(2)$ & M3"-O10d & $2.040(5)$ \\
\hline $\mathrm{Ca}-\mathrm{O} 7$ & $2.546(2)$ & M3"-O13 & $2.447(9)$ \\
\hline $\mathrm{Ca}-\mathrm{O} 9$ & $2.555(2)$ & $<\mathrm{M} 3^{\prime \prime}-\mathrm{O}>$ & 2.285 \\
\hline $\mathrm{Ca}-\mathrm{O} 10$ & 2.695(2) & & \\
\hline $\mathrm{Ca}-\mathrm{O} 11 \mathrm{j}$ & $2.407(2)$ & M3"'-O1d & $2.107(4)$ \\
\hline $\mathrm{Ca}-\mathrm{O} 12 \mathrm{j}$ & $2.738(2)$ & M3"'-O2e & $2.143(5)$ \\
\hline $\mathrm{Ca}-\mathrm{O} 13 \mathrm{~d}$ & $2.659(2)$ & M3"'-O7 & $2.217(8)$ \\
\hline \multirow[t]{2}{*}{$<\mathrm{Ca}-\mathrm{O}>$} & 2.555 & M3"'-O9 & $2.254(7)$ \\
\hline & & M3"'-O10d & $2.456(7)$ \\
\hline M3'-M3" & $0.33(1)$ & M3"'-O13 & $2.017(9)$ \\
\hline M3'-M3"' & $0.32(1)$ & $<\mathrm{M} 3$ "'-O > & 2.199 \\
\hline M3"-M3"' & $0.65(1)$ & & \\
\hline \multirow[t]{2}{*}{$\mathrm{Na}-\mathrm{Nak}$} & $0.49(2)$ & $\mathrm{O} 13-\mathrm{H} 1$ & $0.98(4)$ \\
\hline & & $\mathrm{O} 13-\mathrm{H} 2$ & $0.98(4)$ \\
\hline
\end{tabular}

Note: a: $x,-1 / 2+y, 1 / 2-z ; \mathrm{b}: 1 / 2+x, 1 / 2-y, z ; \mathrm{c}: 1 / 2-x, y,-1 / 2+z ; \mathrm{d}:-1 / 2+x, 1 / 2-y$, $z$; e: $1 / 2-x, y, 1 / 2+z$; f: $1 / 2-x,-1 / 2+y,-z ;$ g: $-1 / 2+x,-y, z$; h: $-x, 1 / 2-y,-1 / 2+z$; $\mathrm{i}:-1 / 2+x,-y, 1 / 2-z ; \mathrm{j}: 1 / 2-x, 1 / 2+y, 1-z ; \mathrm{k}:-x,-y,-z$. polarization and background effects, averaged and reduced to structure factors; of the 2773 unique reflections, 2530 were considered as observed $\left(\left|F_{\mathrm{o}}\right|>5 \sigma\left|F_{\mathrm{o}}\right|\right)$.

Following collection of the X-ray intensity data, the crystal was mounted in epoxy, ground, polished, carbon-coated, and analyzed by an electron microprobe as described above.

\section{STRUCTURE SOLUTION AND REFINEMENT}

All calculations were done with the SHELXTL PC Plus system of programs; $R$ and $w R$ indices are of the conventional form and are given as percentages. The structure was solved by direct methods. The $E$ statistics indicate that the structure is centrosymmetric. The structure was solved and refined in space group $P c a b$ although there are 23 reflections $\left(F_{0}^{2}>5 \sigma F_{0}^{2}\right)$ violating the extinction conditions; the strongest of these violate the $b$ glide. This aspect of the work will be discussed later. The solution with the highest combined figure-of-merit proved to be correct, and the structure was refined by a combination of least-squares refinement and difference-Fourier synthesis to an $R$ index of $2.8 \%$, using soft constraints for the $\mathrm{O}-\mathrm{H}$ distances. The maximum and minimum residual electron-density peaks are 0.88 and $-0.87 / \AA^{3}$, respectively. Final atomic parameters are listed in Table 4, selected interatomic distances are given in Table 5, and refined site-scattering values are listed in Table 6. A bond-valence table, calculated from the parameters of Brown and Altermatt (1985), is shown as Table 7. Observed and calculated structure-factors are listed in Table $8 .{ }^{1}$

${ }^{1}$ For a copy of Table 8, document item AM-99-025, contact the Business Office of the Mineralogical Society of America (see inside front cover of recent issue) for price information. Deposit items may also be available on the American Mineralogist web site (http://www.minsocam.org or current web address). 
TABLE 6. Refined site-scattering values (epfu) and assigned site-populations for bederite

\begin{tabular}{|c|c|c|c|c|c|}
\hline Site & Site scattering & Site population & $\begin{array}{c}\text { Calculated } \\
\text { site-scattering }\end{array}$ & $\begin{array}{c}<M-O>_{\text {obs }} \\
(\AA)\end{array}$ & $\begin{array}{c}<\mathrm{M}-\mathrm{O}\rangle_{\text {calc }} \\
(\AA)\end{array}$ \\
\hline M1 & $21.8(1)$ & $1.01 \mathrm{Mn}^{2+}+0.62 \mathrm{Mg}+0.32 \mathrm{Fe}^{2+}+0.05 \mathrm{Zn}$ & 21.3 & 2.152 & 2.15 \\
\hline M2 & $22.6(1)$ & $1.56 \mathrm{Fe}^{3+}+0.27 \mathrm{Mg}+0.17 \mathrm{Al}$ & 23.0 & 2.029 & 2.01 \\
\hline M3' & 25.4 & $2.00 \mathrm{Mn}^{2+}$ & 25.0 & 2.235 & 2.20 \\
\hline M3" & $12.4(4)$ & $0.49 \mathrm{Mn}^{2+}$ & 12.3 & - & - \\
\hline M3"' & $6.6(5)$ & $0.26 \mathrm{Mn}^{2+}$ & 6.5 & - & - \\
\hline $\mathrm{M} 3 \mathrm{r}$ & $6.4(5)$ & $0.25 \mathrm{Mn}^{2+}$ & 6.3 & - & - \\
\hline $\mathrm{Na}$ & $2.7(1)$ & $0.21 \mathrm{Na}+0.05 \mathrm{Ca}$ & 3.3 & - & - \\
\hline
\end{tabular}

TABLE 7. Bond-valences (v.u.) for bederite*

\begin{tabular}{|c|c|c|c|c|c|c|c|c|c|c|c|}
\hline & P1 & P2 & P3 & M1 & M2 & M3 & $\mathrm{Ca}$ & $\mathrm{Na}$ & $\mathrm{H} 1$ & $\mathrm{H} 2$ & $\Sigma$ \\
\hline$\overline{\mathrm{O} 1}$ & 1.25 & & & 0.42 & & 0.34 & & & & & 2.01 \\
\hline $\mathrm{O} 2$ & 1.27 & & & & & 0.42 & 0.24 & $\begin{array}{l}0.04 \\
0.01\end{array}$ & & & 1.98 \\
\hline O3 & 1.21 & & & 0.36 & 0.43 & & & & & & 2.00 \\
\hline O4 & 1.21 & & & & 0.45 & & 0.25 & & & & 1.91 \\
\hline O5 & & 1.22 & & 0.39 & 0.38 & & & & & & 1.99 \\
\hline O6 & & 1.25 & & 0.41 & & & 0.29 & & & & 1.95 \\
\hline 07 & & 1.25 & & & & 0.42 & 0.21 & $\begin{array}{l}0.11 \\
0.11\end{array}$ & & & 2.10 \\
\hline O8 & & 1.22 & & & 0.55 & & & & 0.20 & 0.20 & 2.17 \\
\hline 09 & & & 1.23 & 0.25 & & 0.16 & 0.20 & & & & 1.84 \\
\hline O10 & & & 1.20 & 0.25 & & 0.33 & 0.14 & & & & 1.92 \\
\hline O11 & & & 1.22 & & 0.45 & & 0.30 & & & & 1.97 \\
\hline O12 & & & 1.21 & & 0.53 & & 0.12 & $\begin{array}{l}0.02 \\
0.05\end{array}$ & & & 1.93 \\
\hline 013 & & & & & & 0.34 & 0.15 & & 0.80 & 0.80 & 2.09 \\
\hline$\Sigma$ & 4.94 & 4.94 & 4.86 & 2.08 & 2.79 & 2.01 & 1.90 & 0.34 & 1.00 & 1.00 & \\
\hline
\end{tabular}

TABLE 9. Site populations in end-member wicksite, bederite, the wicksite-like phase and grischunite

\begin{tabular}{|c|c|c|c|c|c|c|c|}
\hline Site & $\mathrm{Na}$ & $\mathrm{Ca}$ & M1 & M2 & M3 & & \\
\hline $\begin{array}{l}\text { Wicksite } \\
\text { Bederite } \\
\text { Wicksite-like* } \\
\text { Grischunite }\end{array}$ & $\begin{array}{l}\mathrm{Na} \\
\square \\
\square \\
\mathrm{Na}\end{array}$ & $\begin{array}{l}\mathrm{Ca}_{2} \\
\mathrm{Ca}_{2} \\
\mathrm{Ca}_{2} \\
\mathrm{Ca}\end{array}$ & $\begin{array}{l}\mathrm{Fe}_{2}^{2+} \\
\mathrm{Mn}_{2}^{2+} \\
\mathrm{Fe}_{2}^{2+} \\
\mathrm{Mn}_{2}^{2+}\end{array}$ & $\begin{array}{l}{\left[\mathrm{Fe}_{1}^{3+} \mathrm{Mg}_{1}\right]} \\
\mathrm{Fe}_{2}^{3+} \\
\mathrm{Fe}_{2}^{3+} \\
{\left[\mathrm{Fe}_{1}^{3+} \mathrm{Mn}_{1}^{2+}\right]}\end{array}$ & $\begin{array}{l}\mathrm{Fe}_{2}^{2+} \\
\mathrm{Mn}_{2}^{2+} \\
\mathrm{Mn}_{2}^{2+} \\
\mathrm{Mn}_{2}^{2+}\end{array}$ & $\begin{array}{l}\left(\mathrm{PO}_{4}\right)_{6} \\
\left(\mathrm{PO}_{4}\right)_{6} \\
\left(\mathrm{PO}_{4}\right)_{6} \\
\left(\mathrm{AsO}_{4}\right)_{6}\end{array}$ & $\begin{array}{l}\left(\mathrm{H}_{2} \mathrm{O}\right)_{2} \\
\left(\mathrm{H}_{2} \mathrm{O}\right)_{2} \\
\left(\mathrm{H}_{2} \mathrm{O}\right)_{2} \\
\left(\mathrm{H}_{2} \mathrm{O}\right)_{2}\end{array}$ \\
\hline
\end{tabular}

* The occupancies of M1 and M3 are speculative, but reflect the bulk composition of this phase.

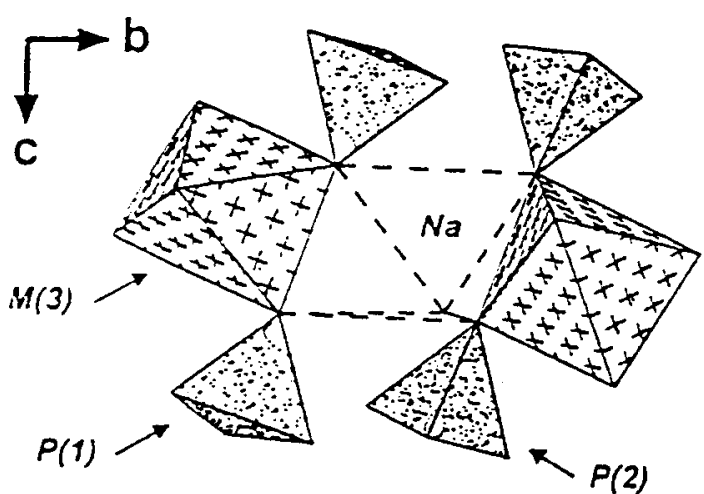

bederite

$$
\begin{aligned}
N a & =\square, \mathrm{Na} \\
M(3) & =\mathrm{Mn}^{2+}
\end{aligned}
$$

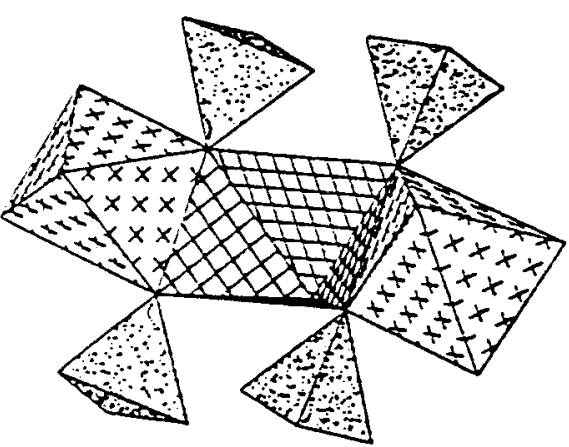

wicksite

$$
\begin{aligned}
\mathrm{Na} & =\mathrm{Na} \\
\mathrm{M}(3) & =\mathrm{Fe}^{2+}, \mathrm{Mn}^{2+}
\end{aligned}
$$

FigURE 1. The local environment of the Na site in the crystal structures of bederite (left) and wicksite (right). The Na octahedron is on the center of symmetry at the origin between the two M3 octahedra (cross-shaded). 


\section{DESCRIPTION OF THE STRUCTURE}

Bederite is isostructural with wicksite, ideally $\mathrm{NaCa}_{2} \mathrm{Fe}_{2}^{2+}$ $\left[\mathrm{Fe}^{3+} \mathrm{Mg}\right] \mathrm{Fe}_{2}^{2+}\left(\mathrm{PO}_{4}\right)_{6}\left(\mathrm{H}_{2} \mathrm{O}\right)_{2}$ (Cooper and Hawthorne 1997) and grischunite, ideally $\mathrm{NaCa}_{2} \mathrm{Mn}_{2}^{2+}\left[\mathrm{Fe}^{3+} \mathrm{Mn}^{2+}\right] \mathrm{Mn}_{2}^{2+}\left(\mathrm{AsO}_{4}\right)_{6}\left(\mathrm{H}_{2} \mathrm{O}\right)_{2}$ (Graeser et al. 1984; Bianchi et al. 1987). There are three octahedrally coordinated $\mathrm{M}$ sites in this structure type, one ninefold-coordinated $\mathrm{Ca}$ site and one sixfold-coordinated $\mathrm{Na}$ site; the populations of these sites in end-member wicksite are given in Table 9. We now assign the site populations in bederite, based on a combination of the results of the site-scattering refinement, the observed mean bond lengths and the unit formula calculated from the electron-microprobe analysis.

\section{The Ca and Na sites}

The scattering and mean bond length at the Ca site are compatible with complete occupancy of this site by $\mathrm{Ca}$, in accord with the unit formula (Table 1). The refined site-scattering at the $\mathrm{Na}$ site is low (Table 6), in line with the small amount of $\mathrm{Na}$ $(+$ minor $\mathrm{Ca})$ in the electron-microprobe analysis. The $\mathrm{Na}$ atom is slightly disordered off the (000) position, presumably in response to disorder at the associated M3 sites (Fig. 1).

\section{The M sites}

The M3 site was actually split into three subsites in the final stages of refinement. However, for the initial assignment of site populations, the M3', M3", and M3'" sites were summed in terms of their refined site-scattering values and mean bond lengths; this aggregate site is designated as M3 (Table 7). The site scattering and mean bond length at $\mathrm{M} 3$ are compatible with complete occupancy of this (aggregate) site by $\mathrm{Mn}^{2+}$; the resultant calculated mean bond length is $0.83+1.37=2.20 \AA$ (radii from Shannon 1976). The subsequent assignment of $\mathrm{Mn}^{2+}$ to the constituent subsites is straightforward, although mean bond lengths cannot be calculated.

The $\langle\mathrm{M} 1-\mathrm{O}\rangle$ and $\langle\mathrm{M} 2-\mathrm{O}\rangle$ distances are 2.152 and $2.029 \AA$, respectively, indicating that the trivalent cations $\mathrm{Fe}^{3+}$ and $\mathrm{Al}$ are ordered at the $\mathrm{M} 2$ site. The $\mathrm{Mg}^{*}(=\mathrm{Mg}+\mathrm{Al})$ and $\mathrm{Fe}^{*}\left(=\mathrm{Fe}^{2+}+\right.$ $\mathrm{Fe}^{3+}+\mathrm{Mn}^{2+}$ ) occupancies can be assigned directly from the refined site-scattering values of Table 6 . As indicated above, if all $\mathrm{Fe}^{*}$ at $\mathrm{M} 2$ is assigned as $\mathrm{Fe}^{3+}$, the resultant sum of the constituent cation and anion radii is close to the observed mean bond length (Table 6). The $\mathrm{Fe}^{*}$ at M1 was assigned as $\mathrm{Mn}^{2+}+\mathrm{Fe}^{2+}$ and $\mathrm{Mg}^{*}$ was assigned as $\mathrm{Mg}$, and the resultant sum of the constituent radii is close to the observed mean bond length. The formula was recalculated with the $\mathrm{Fe}^{3+} / \mathrm{Fe}^{2+}$ ratio derived from these site occupancies, and the resultant unit formula and assigned site populations are given in Tables 1 and 6, respectively.

\section{COMPARISON OF BEDERITE AND WICKSITE}

The end-member formulae of wicksite, bederite, the unfortunate wicksite-like phase and grischunite are given in Table 9. The principal differences between the three phases are (1) in wicksite, the $\mathrm{Na}$ site is occupied by $\mathrm{Na}$ whereas it is vacant in bederite and the wicksite-like phase; (2) the M1 and M3 sites are occupied by $\mathrm{Fe}^{2+}$ in wicksite, $\mathrm{Mn}^{2+}$ in bederite and $\mathrm{Mn}^{2+}+$ $\mathrm{Fe}^{2+}$ ) in the wicksite-like phase; (3) the M2 site is occupied by
$\mathrm{Fe}^{3+} \mathrm{Mg}$ in wicksite, whereas it is occupied by $\mathrm{Fe}^{+}$in bederite and the wicksite-like phase.

Thus bederite is related to wicksite by the substitutions

$$
\begin{gathered}
\mathrm{Na} \square+{ }^{\mathrm{M} 2} \mathrm{Fe}^{3+} \rightarrow{ }^{\mathrm{Na}} \mathrm{Na}+{ }^{\mathrm{M} 2} \mathrm{Mg} \\
{ }^{\mathrm{M} 1} \mathrm{Mn}^{2+} \rightarrow{ }^{\mathrm{M} 1} \mathrm{Fe}^{2+} \\
{ }^{\mathrm{M} 3} \mathrm{Mn}^{2+} \rightarrow{ }^{\mathrm{M} 3} \mathrm{Fe}^{2+}
\end{gathered}
$$

Substitution 1 is heterovalent and hence justifies a new root name. Substitutions 2 and 3 alone would just produce the Mnequivalent of wicksite, but the substitution of a vacancy $(\square)$ for $\mathrm{Na}$ at the $\mathrm{Na}$ site (balanced by the substitution of $\mathrm{Fe}^{3+}$ for $\mathrm{Mg}$ at the M2 site) requires a new name.

Bederite shows 23 reflections violating Pcab symmetry. Cooper and Hawthorne (1997) noted that in wicksite and grischunite, the M2 site is occupied by equal amounts of divalent and trivalent cations, suggesting the possibility of lower symmetry. Moreover, Sturman et al. (1981) reported that wicksite shows signs of anomalous optics. However, Cooper and Hawthorne (1997) were unable to satisfactorily refine the structures of wicksite or grischunite in monoclinic symmetry. The diffraction characteristics of bederite also suggest lower symmetry for the minerals of this group, even though the structures can be well-refined in orthorhombic symmetry.

\section{ACKNOWLEDGMENTS}

This work was done during the sabbatical leave of M. Galliski as Visiting Researcher at the University of Manitoba, and during a later short visit facilitated by a grant of the Fundación Antorchas, which is gratefully acknowledged. Financial support was provided by Natural Sciences and Engineering Research Council of Canada Grants to F.C.H. and P.C.

\section{REFERENCES CITED}

Bianchi, R., Pilati, T., and Mannucci, G. (1987) Crystal structure of grischunite. American Mineralogist, 72, 1225-1229.

Brown, I.D. and Altermatt, D. (1985) Bond-valence parameters obtained from a systematic analysis of the inorganic crystal structure database. Acta Crystallographica, B41, 244-247.

Cooper, M.A. and Hawthorne, F.C. (1997) The crystal structure of wicksite. Canadian Mineralogist, 35, 777-784.

Galliski, M.A. (1983a) Distrito Minero El Quemado, Departamentos La Poma y Cachi, Provincia de Salta. I: El basamento del tramo septentrional de la Sierra de Cachi. Revista Asociación Geológica Argentina, 38, 209-224.

(1983b) Distrito Minero El Quemado, Departamentos La Poma y Cachi, Provincia de Salta. II: Geologia de sus Pegmatitas. Revista Asociación Geológica Argentina, 38, 340-380.

Galliski, M.A. and Sureda, R.J. (1982) Presentaciones y propiedades de Alluaudita en el Distrito Minero El Quemado, Provincia de Salta, Argentina, Actas V Congreso Latinoamericano de Geologia, IV, 401-408.

Graeser, S., Schwander, H., and Suhner, B. (1984) Grischunit $\mathrm{CaMn}_{2}\left(\mathrm{AsO}_{4}\right)_{2}$, eine neue Mineralart aus den Schweizer Alpen. Schweizerische Mineralogische und Petrographische Mitteilungen, 64, 1-10.

Peacor, D.R., Dunn, P.J., Ramik, R.A., Campbell, T.J., and Roberts, W.L. (1985) A wicksite-like mineral from the Bull Moose mine, South Dakota. Canadian Mineralogist, 23, 247-249.

Pouchou, J. and Pichoir, F. (1985) 'PAP' $\phi(\rho Z)$ procedure for improved quantitative microanalysis. Microbeam Analysis 1985, San Francisco Press, California.

Shannon, R.D. (1976) Revised effective ionic radii and systematic studies of interatomic distances in halides and chalcogenides. Acta Crystallographica, A32, 751-767.

Sturman, B.D., Peacor, D.R., and Dunn, P.J. (1981) Wicksite, a new mineral from Northeastern Yukon Territory. Canadian Mineralogist, 19, 377-380.

MANUSCRIPT RECEIVED AUGUST 31, 1998 MANUSCRIPT ACCEPTED JUNE 13, 1999 PAPER HANDLED BY SIMON A.T. REDFERN 